\title{
Cooperative and Competitive Dynamics Model for Information Propagation in Online Social Networks
}

\author{
Yaming Zhang, ${ }^{1}$ Chaosheng Tang, ${ }^{1}$ and Li Weigang ${ }^{2}$ \\ ${ }^{1}$ School of Economics and Management, Yanshan University, Qinhuangdao 066004, China \\ ${ }^{2}$ Department of Computer Science, University of Brasilia, 70910-900 Brasilia, DF, Brazil \\ Correspondence should be addressed to Yaming Zhang; yaming99@ysu.edu.cn
}

Received 1 May 2014; Revised 23 June 2014; Accepted 23 June 2014; Published 13 July 2014

Academic Editor: Francisco J. Marcellán

Copyright (c) 2014 Yaming Zhang et al. This is an open access article distributed under the Creative Commons Attribution License, which permits unrestricted use, distribution, and reproduction in any medium, provided the original work is properly cited.

\begin{abstract}
Traditional empirical models of propagation consider individual contagion as an independent process, thus spreading in isolation manner. In this paper, we study how different contagions interact with each other as they spread through the network in order to propose an alternative dynamics model for information propagation. The proposed model is a novel combination of Lotka-Volterra cooperative model and competitive model. It is assumed that the interaction of one message on another is flexible instead of always negative. We prove that the impact of competition depends on the critical speed of the messages. By analyzing the differential equations, one or two stable equilibrium points can be found under certain conditions. Simulation results not only show the correctness of our theoretical analyses but also provide a more attractive conclusion. Different types of messages could coexist in the condition of high critical speed and intense competitive environment, or vice versa. The messages will benefit from the high critical speed when they are both competitive, and adopting a Tit-for-Tat strategy is necessary during the process of information propagation.
\end{abstract}

\section{Introduction}

With the rapid development of information technology and the widespread use of intelligent communication tools, we progress further into the age of information explosion and have been bombarded with massive amounts of data. For instance, there are more than 368 million users in Sina Weibo and 100 million messages are distributed every day [1], while over 500 million registered users and more than 200 million tweets are posted every signal day in Twitter [2]. As the Twitter and other online social networking (OSN) applications provide great facility by their convenient and efficient way of information dissemination, various studies focus on the issue of many complex phenomena during the process of the spreading of information over networks. Because of the complexity of the system, OSN is generally characterized as being complex networks, in which individuals are represented by nodes, and information interactions between individuals are represented by links between nodes [3]. Since the propagation of information and spreading of epidemic infection have a lot in common, many epidemic models have been used to describe and explore the evolution process of information. For instance, the process of rumor propagation can be viewed as a process of the individuals being infected. After being infected, the ignorants become spreader with a probability, and then the spreaders can become the stiflers, which is similar as the infected persons can recover over a period of time [4].

Many researches have been devoted to the propagation dynamics. The significant model for information or rumor spreading was introduced by Daley and Kendall many years ago $[5,6]$. Other variants based on Daley-Kendall (DK) model, such as Maki-Thompson (MK) model [7], have been widely used in the past for quantitative study of the mechanism of information dissemination. In DK model, a closed and homogeneously mixed population is classified into three main groups: ignorants, spreaders, and stiflers. Ignorants are susceptible to rumor or other information. Spreaders play an important role in accelerating information diffusion. Stiflers are the individuals who get tired of rumors and refuse to propagate them [8]. In DK model, the rumor is propagated through the population by pairwise contacts 
between spreaders and others, while it is spread by directed contacts of the spreaders with others in MK model.

As the continuous development of research on complex networks, a number of recent studies confirm that the network topology has a marked influence on the whole diffusion process. In the implementation of the MK model, Zanette [9] showed that the simulation results exhibited a transition between regimes of localization and propagation at a finite value of the network randomness. Moreno et al. [10] studied the dynamics of epidemic spreading processes by Monte Carlo simulations and gave a numerical solution of rumor diffusion mean-field equations [11]. Zhou et al. [12] focused on the influence of network structure on rumor propagation and concluded that the total final infected nodes will decrease when the structure changes from random to scale-free network. Some studies described a formulation of the DK model on complex networks in terms of interacting Markov chains. Nekovee et al. [13] derived mean-field equations for the dynamics of rumor spreading on the arbitrary degree correlated networks and drew a conclusion that scalefree social networks are prone to the spreading of rumors. These studies revealed a complex interplay between the network topology and found that the final infected density of population with degree $k$ is related to the network structure. When the structure changes from random networks to scalefree networks, the rumor is more easily spread.

Besides the epidemic dynamics models mentioned above, other widely used models describing information propagation are diffusion models in social networks. For instance, the threshold models developed by Granovetter [14] described collective social behavior, and the simplest version of linear threshold model presented by Kempe et al. [15] has taken social network structure into account during the process of information propagation. Those models characterized the information propagation based on a directed graph which originated from social networks, and the inactive nodes could turn into active ones with a certain probability under the influence of a small number of initially activated nodes.

Although extensive progress has been made in understanding the dynamics of the information spreading, most studies focused on one type of information or topic through a complex network in the context of dynamics of information spreading. Only a few of unsystematic studies concern the case that more than one type of information coexists and spreads among the population, such as two rumors with different probabilities of acceptance spreading among nodes [16], two types of information spreading among a population of individuals [17], competing ideas in complex social systems [18], and the propagation of competing memes on composite networks $[19,20]$.

Nevertheless, all of these studies explored different contagions competing with each other as they spread over the network but neglected the phenomena of the cooperation as they mutually help each other in spreading. In fact, there are multiple pieces of information about an event spreading through the OSN simultaneously. Moreover, these pieces of information do not spread in isolation manner from all other information currently diffusing in the network. The built-in mechanism underlying these complex interactions between different types of contagions makes a noticeable impact on dynamic spreading. For instance, competing contagions decrease each other's probability of spreading, while cooperating contagions help each other in being adopted throughout the network [21]. Thus, it is necessary for us to model the interaction between contagions and not just consider each contagion in isolation manner separated from others.

As the main contribution of this paper, we propose a cooperative and competitive model for the different contagions of information diffusion in OSN. First of all, the proposed model is based on the population dynamics models to describe the development tendency and interaction between two different types of information. It provides a more realistic description of this process comparing with previous models by Lotka-Volterra and others. Secondly, we use differential equations of stability theory to analyze the proposed model. By means of the approximate analytical and exact numerical solutions of these equations, we examine the steady-state of information propagation in OSN. Finally, the analytic results with the real data collected from Sina Weibo indicate that the proposed model is effective for understanding and explaining some social phenomena presented in the process of online information spreading, especially the vital role of the cooperation and competition in the information propagation.

The rest of this paper is organized as follows. In Section 2 a formulation of the information spreading model within the framework of biological models is proposed. Section 3 is devoted to analyzing the cooperative and competitive model and explaining the results of the steady-state behavior. In Section 4, the numerical simulation with the data from Sina Weibo demonstrates the investigations of the steady state and dynamics of the model. The last section concludes the paper with the direction of further research.

\section{Model}

In this section, we describe the basic information spreading system and then establish a general co-competition model based on Lotka-Volterra model.

2.1. System Description. In OSNs websites, once a user publishes a message on Twitter or other microblog websites, the message will be transmitted to his/her followers. If the message is so interesting that some followers decide to repost it [3], this message can be read by more users and the information could thus reach beyond the network of the original author. We define the user behaviors, such as retweet, review, and mention, as a growth factor. The greater this value is, the faster the message spreads, and more users see the message. Meanwhile, the individuals may also cease spreading a message spontaneously at a certain rate influenced by time decaying effect [22]. This phenomenon can be considered as decay factor of the message.

Considering the special time attributes of the information spreading in OSNs, we choose the user growth rate $v(t)$ as dynamical variables instead of the users themselves. In OSNs, the spread of messages is primarily determined by the repost number in time interval. For example, when we describe 
the spread of a tweet in Twitter, it is usually defined that this message spread more than $N$ times per hour, or the message has been reposted a total number of nearly $N$ times by time $t$. However, the latter is a cumulative value that cannot reflect a certain number of changes at some points. Furthermore, the message spread process has strong timeliness and variation in OSNs, and each message has a life cycle that spreads slowly at beginning and then breaks out and vanishes finally. The variables $v(t)$ can reflect the life circle curve of the message while the variables $N$ cannot.

Assume that there is a message published at time $t$ in Twitter, then users repost this tweet and the message could spread at speed $v(t)$. As mentioned above, we set $\mu(v)$ as a function of the speed $v(t)$. However, the speed could not immeasurably increase, and there should be a maximum value $V$. When the speed $v(t)$ achieves $V$, its value will decrease. We suppose that the spread of messages obeys logistic growth pattern in OSNs and set

$$
\mu(v)=\delta \cdot\left(1-\frac{v(t)}{V}\right) .
$$

Here $\delta$ is a constant, denoting the growth factor influence on speed. The speed $v(t)$ can be denoted as $v(t)=d N(t) / d t$, $N(t)$ denotes the total number of people who have heard the message up to time $t$, and $v(t)$ denote the variation number in time interval $[t-1, t]$. Meanwhile, the spread of message in OSNs will be influenced by growth factor $\theta(t)$ and decay factor $\lambda$; we can establish an equation to describe this dynamic process

$$
\frac{d v(t)}{d t}=\delta \cdot\left(1-\frac{v(t)}{V}\right) \cdot \theta(t)-\lambda v(t) .
$$

We discuss the main features of (2) as follows.

(i) When $\theta(t)=0$ or the speed $v(t)$ reaches its maximum value $V$, (2) becomes

$$
\frac{d v(t)}{d t}=-\lambda v(t)
$$

In (3), $v(t)$ is a reduction function that the speed will decrease with time.

(ii) When $\theta(t)$ is a nonzero constant, we introduce variables $\gamma$ and $\kappa$; set

$$
\begin{gathered}
\gamma=\frac{\delta \cdot \theta(t)}{V}+\lambda \\
\kappa=\delta \cdot \theta(t)
\end{gathered}
$$

then (2) becomes

$$
\frac{d v(t)}{d t}=\kappa-\gamma v(t)
$$

Set $v(0)=v_{0}$,

$$
v(t)=\frac{\kappa}{\gamma}\left(1-e^{-\gamma t}\right)+v_{0} e^{-\gamma t}
$$

Equation (8) denotes the function of $v(t)$ when $\theta(t)$ is a nonzero constant. (iii) When the speed remains unchanged, $d v(t) / d t=0$, (2) becomes

$$
\delta \cdot\left(1-\frac{v(t)}{V}\right) \cdot \theta(t)-\lambda v(t)=0
$$

then we obtain

$$
v(t)=\frac{\delta V \theta(t)}{\delta \theta(t)+\lambda V} .
$$

Based on the above analysis, we prove that the speed $v(t)$ is a function of the growth factor $\theta(t)$. In next section, we further discuss the situation that different types of messages are spreading over networks.

2.2. Co-Competition Lotka-Volterra Model. The spreading of abundance of information to which we are exposed through online social networks is a complex sociopsychological process. In the real world, these contagions not only propagate at the same time but also interplay with each other as they spread over the networks. This phenomenon is similar to the mutualism-competition interaction among multiple species. During the spreading of pieces of information, the cooperation happens when the different types of information are at low speed, and the competition happens when they are at high speed. Hence, according to [23], the general cooperation-competition model of $n$ types of information can be presented as follows: $d v_{i} / d t=v_{i} f_{i}\left(v_{1}, v_{2}, \ldots, v_{n}\right)$, where $v_{i}$ denotes the speed of the $i$ th messages and $f_{i}\left(v_{1}, v_{2}, \ldots, v_{n}\right)$ represents its growth rate. The partial derivatives of the function $f_{i}\left(v_{1}, v_{2}, \ldots, v_{n}\right)$ can be written as $\partial f_{i} / \partial v_{j}, i, j=$ $1,2, \ldots, n$. The cooperation-competition interaction is set by (9) by satisfying the condition that for each $i, 1 \leq i \leq n$, there is $b_{i}>0$. Consider

$$
\begin{gathered}
\frac{\partial f_{j}}{\partial v_{i}}>0, \quad 0<v_{i}<b_{i}, \\
\frac{\partial f_{j}}{\partial v_{i}}<0, \quad v_{i}>b_{i},
\end{gathered}
$$

where $i \neq j, i, j=1,2, \ldots, n$. According to the ecological theory, we can infer that the $i$ th message has a positive effect on other messages as it is at low density $\left(0<v_{i}<b_{i}\right)$, while it has negative effect on them at high density $\left(v_{i}>b_{i}\right)$.

We study the common case that two different types of information interact with each other and then make a natural extension of the classical two-species Lotka-Volterra competitive model and Lotka-Volterra cooperative model. We assume that the spread of each message is affected by the internal attractiveness itself and the external pressure among messages simultaneously. Considering two different types of information on the same event spreading around the same time in Twitter or other OSNs, they will compete with each other for the reason that users only choose to believe one of them. In the meantime, if all the messages are so important or interesting, users would be more likely to adopt and share them. It is considered that they mutually help each other in spreading through the network. We term the two types 
of information as message 1 and message 2, and they will interact with each other when they spread at the same time. The propagation process and the interactions between the two types of information can be governed by the following set of rules.

(i) Each message has a relatively stable natural growth coefficient $\mu_{i}$ and a decay coefficient $\lambda_{i}$, which represent the growth factor and decay factor, respectively, and $\mu_{i}>\lambda_{i}$.

(ii) Since a signal message could not spread indefinitely, there exists a maximum speed $V_{i}$ during the propagation process.

(iii) Two messages will compete or cooperate with one another and take negative or positive effect, represented by $\sigma_{i j}$.

Supported by the rules (i) and (ii), we have the following model:

$$
\begin{aligned}
& \frac{d v_{1}(t)}{d t}=\mu_{1} v_{1}\left(1-\frac{v_{1}}{V_{1}}\right)-\lambda_{1} v_{1}, \\
& \frac{d v_{2}(t)}{d t}=\mu_{2} v_{2}\left(1-\frac{v_{2}}{V_{2}}\right)-\lambda_{2} v_{2} .
\end{aligned}
$$

Here $\mu_{i}$ denotes the growth coefficient of the $i$ th message and $\mu_{i} \in[0,1] ; \lambda_{i}$ denotes the decay coefficient of the $i$ th message and $\lambda_{i} \in[0,1], i=1,2$.

According to the rule (iii), when the messages 1 and 2 coexist in the network, the interactive influence among the two messages produces positive effect or negative effect as they cooperate or compete with each other. Based on the analysis above, (10) can be written as

$$
\begin{aligned}
& \frac{d v_{1}(t)}{d t}=\mu_{1} v_{1}\left(1+\sigma_{21} \frac{\beta}{V_{2}}-\frac{v_{1}}{V_{1}}-\sigma_{21} \frac{\left|v_{2}-\beta\right|^{*}}{V_{2}}\right)-\lambda_{1} v_{1}, \\
& \frac{d v_{2}(t)}{d t}=\mu_{2} v_{2}\left(1+\sigma_{12} \frac{\alpha}{V_{1}}-\frac{v_{2}}{V_{2}}-\sigma_{12} \frac{\left|v_{1}-\alpha\right|^{*}}{V_{1}}\right)-\lambda_{2} v_{2} .
\end{aligned}
$$

In order to facilitate the analysis, set $a=1-\lambda_{1} / \mu_{1}, b=$ $1-\lambda_{2} / \mu_{2} ;(11)$ can be written as

$$
\begin{aligned}
& \frac{d v_{1}(t)}{d t}=\mu_{1} v_{1}\left(a+\sigma_{21} \frac{\beta}{V_{2}}-\frac{v_{1}}{V_{1}}-\sigma_{21} \frac{\left|v_{2}-\beta\right|^{*}}{V_{2}}\right), \\
& \frac{d v_{2}(t)}{d t}=\mu_{2} v_{2}\left(b+\sigma_{12} \frac{\alpha}{V_{1}}-\frac{v_{2}}{V_{2}}-\sigma_{12} \frac{\left|v_{1}-\alpha\right|^{*}}{V_{1}}\right) .
\end{aligned}
$$

Equation (12) can be considered as a competition and cooperation multisystem. Let $v_{2}=0$ in the first equation of the system (12); we have $d v_{1} / d t=\mu_{1} v_{1}\left(a V_{1}-v_{1}\right) / V_{1}$, then the parameter $a V_{1}$ is the speed of the message 1 when it disseminates independently form message 2. Let $C_{1}\left(v_{1}, v_{2}\right)=$ $a+\sigma_{21}\left(\beta / V_{2}\right)-v_{1} / V_{1}-\sigma_{21}\left(\left|v_{2}-\beta\right|^{*} / V_{2}\right)$; the function $\left|v_{2}-\beta\right|^{*}$ can be defined as the absolute function $\left|v_{2}-\beta\right|$ while the function $\left|v_{2}-\beta\right|$ is smoothed in a very small neighborhood

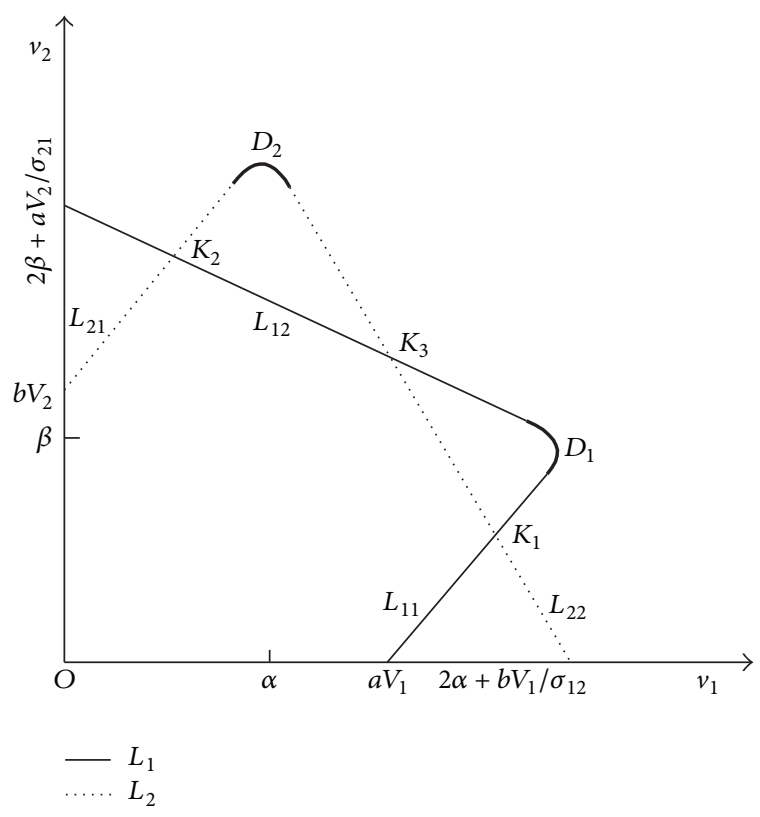

FIGURE 1: The smoothed parts of the isoclines $L_{1}$ and $L_{2}$.

of its vertex $(0, \beta)$. Thus, the isocline $L_{1}: C_{1}\left(v_{1}, v_{2}\right)=0$ is smooth as shown in Figure 1. Then the function $f_{1}\left(v_{1}, v_{2}\right)=$ $\mu_{1} v_{1} C_{1}\left(v_{1}, v_{2}\right)$ and satisfies

$$
\begin{gathered}
\frac{\partial f_{1}}{\partial v_{2}}=\frac{\sigma_{21}}{V_{2}} \mu_{1} v_{1}, \quad 0<v_{2}<\beta, \\
\frac{\partial f_{1}}{\partial v_{2}}=-\frac{\sigma_{21}}{V_{2}} \mu_{1} v_{1}, \quad v_{2}>\beta .
\end{gathered}
$$

As shown in (13), the positive or negative effect acting on message 1 depends on the speed of message 2 . When message 2 travels slowly $\left(v_{2}<\beta\right)$, it takes positive effect on message 1; otherwise, it produces negative effect on message 1 at high speed $\left(v_{2}>\beta\right)$. The parameter $\beta$ signifies the critical speed of message 2 near which the effect of message 2 acting on message 1 would turn positive effect into negative effect. Furthermore, according to the Lotka-Volterra models, the parameter $\sigma_{21}$ represents both the cooperation (at low speed) and competition (at high speed) level of message 2 to message 1 . Hence, the low speed of message $2,0<v_{2}<$ $\beta$, represents the scope of cooperation of message 2 with message 1. A similar conclusion can be obtained that the low speed of message $1,0<v_{1}<\alpha$, represents the region of net cooperation message 1 with message 2 . The isocline $L_{2}: C_{2}\left(v_{1}, v_{2}\right)=b+\sigma_{12}\left(\alpha / V_{1}\right)-v_{2} / V_{2}-\sigma_{12}\left(\left|v_{1}-\alpha\right|^{*} / V_{1}\right)=0$ is smooth as shown in Figure 1.

Since the system (12) can exhibit the features of the LotkaVolterra cooperative model and competitive model, it can be named co-competition Lotka-Volterra model. Obviously, when the messages are at low speed, for instance, $v_{1}<\alpha$ 

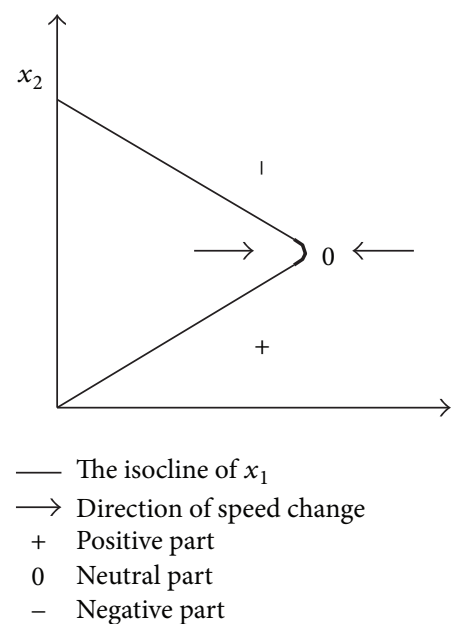

(a)

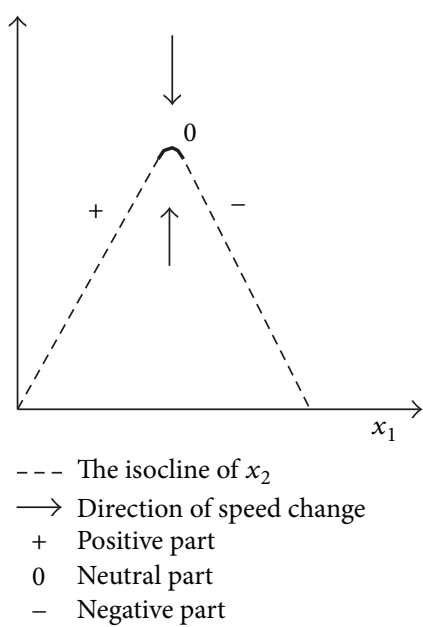

(b)

Figure 2: The parabolic zero growth isoclines of message 1 (a) and message 2 (b).

and $v_{2}<\beta$, the system (12) turns into the Lotka-Volterra cooperative system

$$
\begin{aligned}
& \frac{d v_{1}(t)}{d t}=\mu_{1} v_{1}\left(a+\sigma_{21} \frac{v_{2}}{V_{2}}-\frac{v_{1}}{V_{1}}\right), \\
& \frac{d v_{2}(t)}{d t}=\mu_{2} v_{2}\left(b+\sigma_{12} \frac{v_{1}}{V_{1}}-\frac{v_{2}}{V_{2}}\right) .
\end{aligned}
$$

Conversely, when the messages are at high speed, for instance, $v_{1}>\alpha$ and $v_{2}>\beta$, the system (12) turns into the Lotka-Volterra competitive model

$$
\begin{aligned}
& \frac{d v_{1}(t)}{d t}=\mu_{1} v_{1}\left(a+2 \sigma_{21} \frac{\beta}{V_{2}}-\frac{v_{1}}{V_{1}}-\sigma_{21} \frac{v_{2}}{V_{2}}\right), \\
& \frac{d v_{2}(t)}{d t}=\mu_{2} v_{2}\left(b+2 \sigma_{12} \frac{\alpha}{V_{1}}-\frac{v_{2}}{V_{2}}-\sigma_{12} \frac{v_{1}}{V_{1}}\right) .
\end{aligned}
$$

By systematic comparison of the system (12) with the Lotka-Volterra competitive system (15), an important conclusion is reached that the cooperation at low speed promotes the coexistence level of the two messages in co-opetition LotkaVolterra model. When message 2 is at a low speed $\left(v_{2}<\beta\right)$, it follows the first equation of system (15) that the effect of message 2 acting on message 1 is negative $\left(-\sigma_{21} v_{2} / V_{2}<0\right)$ while it follows the first equation of system (12) that the effect of message 2 acting on message 1 is positive $\left(\sigma_{21} v_{2} / V_{2}>0\right)$, which originates from the cooperation of message 2 at low speed. As a consequence, message 1 has survived for a long time when message 2 is at low speed. When message 2 is at high speed $\left(v_{2}>\beta\right)$, the negative effect produced by message 2 acting on message 1 in system (15) is $\left(-\sigma_{21} v_{2} / V_{2}\right)$ while that in system (12) is $-\sigma_{21}\left(v_{2}-\beta\right) / V_{2}$. Since $-\sigma_{21}\left(v_{2}-\beta\right) / V_{2}>$ $-\sigma_{21} v_{2} / V_{2}$, the negative effect is lessened for the cooperation among messages in system (12). Similarly, the cooperation in system (12) promotes the existence of message 1. In a word, the cooperation in system (12) facilitates the coexistence of both the two messages.

\section{Model Analysis}

In this section, we discuss the intersection of two isoclines $L_{1}$ and $L_{2}$ by a symmetrical hypothesis and then analyze the dynamics of the model and the global stability of the system.

3.1. Basic Analysis. In order to show the existence of intersection of two isoclines $L_{1}$ and $L_{2}$, we assume that the positive or negative effects produced by messages 1 and 2 are almost the same as each other. In the condition, we can set hypothesis as follows: $a=b=z, \sigma_{21}=\sigma_{12}=\sigma, V_{1}=V_{2}=V$, and $\alpha=\beta=\zeta$, then the values of $\mu_{1}$ and $\mu_{2}$ can reflect the difference of the growth factors among the two messages. For $\left|v_{1}-\alpha\right|$ and $\left|v_{2}-\beta\right|$ are smoothed in a very small neighborhood of their vertexes $(0, \alpha)$ and $(0, \beta)$, respectively, the system (12) can be written as

$$
\begin{aligned}
& \frac{d v_{1}(t)}{d t}=\mu_{1} v_{1}\left(z+\sigma \frac{\zeta}{V}-\frac{v_{1}}{V}-\frac{\sigma \zeta}{V}\left|\frac{v_{2}}{\zeta}-1\right|\right), \\
& \frac{d v_{2}(t)}{d t}=\mu_{2} v_{2}\left(z+\sigma \frac{\zeta}{V}-\frac{v_{2}}{V}-\frac{\sigma \zeta}{V}\left|\frac{v_{1}}{\zeta}-1\right|\right) ;
\end{aligned}
$$

set $x_{1}=v_{1} / \zeta$ and $x_{2}=v_{2} / \zeta$; then

$$
\begin{aligned}
& \frac{d x_{1}}{d t}=\mu_{1} v_{1}\left(z+\sigma \frac{\zeta}{V}-\frac{\zeta x_{1}}{V}-\frac{\sigma \zeta}{V}\left|x_{2}-1\right|\right), \\
& \frac{d x_{2}}{d t}=\mu_{2} v_{2}\left(z+\sigma \frac{\zeta}{V}-\frac{\zeta x_{2}}{V}-\frac{\sigma \zeta}{V}\left|x_{1}-1\right|\right) .
\end{aligned}
$$

In int $R_{+}^{2}$, the zero growth isoclines of $x_{1}$ and $x_{2}$ are as follows:

$$
\begin{aligned}
& x_{1}=-\sigma\left|x_{2}-1\right|+C \\
& x_{2}=-\sigma\left|x_{1}-1\right|+C,
\end{aligned}
$$

where $C=V z / \zeta+\sigma$.

According to [24], the isoclines include three parts as shown in Figure 2: positive part (+), neutral part (0), and 
negative part (-). The positive part, neutral part, or negative part is the part of isoclines when the slope (for message 1 , the slope is $d v_{1}(t) / d v_{2}(t)$; for message 2 , the slope is $\left.d v_{2}(t) / d v_{1}(t)\right)$ is positive part $(+)$, neutral part $(0)$, and negative part (-). The two isoclines are symmetry around the line $x_{1}=x_{2}$. Then, we only need to discuss

$$
\begin{aligned}
& x_{1}=x_{2}, \\
& x_{2}=-\sigma\left|x_{1}-1\right|+C .
\end{aligned}
$$

Obviously, (19) always have solutions; we can also obverse the following aspects.

(i) There are two different roots $(\sigma-C) /(\sigma-1)$ and $(\sigma+$ $C) /(\sigma+1)$, when $\sigma^{2} \neq 0,1$ and $C \neq 1$.

(ii) There is double root $x=1$ or $x=C$, when $\sigma^{2} \neq 1$ and $C=1$, or $\sigma=0$.

(iii) There is root $(\sigma-C) /(\sigma-1)$ or $(\sigma+C) /(\sigma+1)$, when $\sigma^{2}=1$ and $C \neq 1$.

Based on the above analysis, we can conclude that trajectories in phase space always intersect isoclines either in the horizontal or in the vertical direction. In the next section, we make further analysis about the stability of intersection.

3.2. Equilibrium. In order to study the final results of interactive effect between two different types of messages, we need to conduct a comprehensive analysis for the stability of the equilibrium points of simultaneous differential equations.

Theorem 1. System (12) admits no periodic orbit.

Proof. Let

$$
\begin{gathered}
B\left(v_{1}, v_{2}\right)=\frac{1}{v_{1} v_{2}} \\
\left(v_{1}, v_{2}\right) \in \operatorname{int} R_{+}^{2}
\end{gathered}
$$

then we have

$$
\begin{aligned}
\frac{\partial}{\partial v_{1}} & {\left[\mu_{1}\left(a+\sigma_{21} \frac{\beta}{V_{2}}-\frac{v_{1}}{V_{1}}-\sigma_{21} \frac{\left|v_{2}-\beta\right|^{*}}{V_{2}}\right)\right] } \\
& +\frac{\partial}{\partial v_{2}}\left[\mu_{2}\left(b+\sigma_{12} \frac{\alpha}{V_{1}}-\frac{v_{2}}{V_{2}}-\sigma_{12} \frac{\left|v_{1}-\alpha\right|^{*}}{V_{1}}\right)\right] \\
= & -\frac{\mu_{1}}{v_{2} V_{1}}-\frac{\mu_{2}}{v_{1} V_{2}}<0 .
\end{aligned}
$$

According to the Bendixson-Dulac theorem [25], system (12) admits no periodic orbit.

We study the evolution of $v_{1}(t)$ and $v_{2}(t)$ as time $t$ approaches infinity. As shown in Figure 1, there are three equilibrium points in system (12): $O_{1}(0,0), O_{2}\left(a V_{1}, 0\right)$, and $\mathrm{O}_{3}\left(0, b V_{2}\right)$. To determine the stability of the equilibrium points, let

$$
M=\left[\begin{array}{ll}
f_{v_{1}} & f_{v 2} \\
g_{v_{1}} & g_{v_{2}}
\end{array}\right]
$$

we have

$$
p=-\left.\left(f_{v_{1}}+g_{v 2}\right)\right|_{p_{i}}, \quad q=\left.\operatorname{det} M\right|_{p_{i}} .
$$

Then, the stability conditions of equilibrium point $O_{i}$ are $p>0$ and $q>0$. The Jacobin matrix of the system (12) at equilibrium state is as follows.

(i) For the equilibrium point $O_{1}(0,0)$, we get

$$
\begin{gathered}
M\left(O_{1}\right)=\left[\begin{array}{cc}
a \mu_{1} & 0 \\
0 & b \mu_{2}
\end{array}\right] \\
p_{1}=-\left(a \mu_{1}+b \mu_{2}\right), \quad q_{1}=a b \mu_{1} \mu_{2} .
\end{gathered}
$$

Based on the assumption that $a=1-\lambda_{1} / \mu_{1}>0$ and $b=$ $1-\lambda_{2} / \mu_{2}>0$, we have $p_{1}<0$ and $q_{1}>0$; the point $O_{1}(0,0)$ is an unstable node.

Since our aim is to show the coexistence of competition and cooperation by the introduction of $\alpha$ and $\beta$, we suppose that the condition (25) is always satisfied in the rest of the paper. Consider

$$
\begin{aligned}
& b+\sigma_{12}\left(\frac{2 \alpha}{V_{1}}-a\right)>0 \\
& a+\sigma_{21}\left(\frac{2 \beta}{V_{2}}-b\right)>0 .
\end{aligned}
$$

(ii) For the equilibrium point $O_{2}\left(a V_{1}, 0\right)$, we get

$$
\begin{aligned}
M\left(O_{2}\right) & =\left[\begin{array}{cc}
-a \mu_{1} & \frac{a V_{1} \mu_{1} \sigma_{21}}{V_{2}} \\
0 & \mu_{2}\left[b+\left(\frac{2 \alpha}{V_{1}}-a\right) \sigma_{12}\right]
\end{array}\right], \\
p_{2} & =a \mu_{1}-\mu_{2}\left[\sigma_{12}\left(\frac{2 \alpha}{V_{1}}-a\right)+b\right] \\
q_{2} & =\frac{a \mu_{1} \mu_{2}\left(-2 \alpha \sigma_{12}+a \sigma_{12} V_{1}-b V_{1}\right)}{V_{1}} .
\end{aligned}
$$

The stability condition of equilibrium point $\mathrm{O}_{2}\left(a V_{1}, 0\right)$ is $\sigma_{12}>b V_{1} /\left(a V_{1}-2 \alpha\right)$.

Condition $\sigma_{12}>b V_{1} /\left(a V_{1}-2 \alpha\right)$ means that message 1 is more competitive than message 2 . It implies that the persons who should have adopted message 2 choose to believe message 1 . Consequently, message 2 will eventually become extinct in the process of competition, while message 1 can continue to diffuse and reach the maximum speed $V_{1}$.

(iii) For the equilibrium point $\mathrm{O}_{3}\left(0, b V_{2}\right)$, we get

$$
\begin{gathered}
M\left(O_{3}\right)=\left[\begin{array}{cc}
\mu_{1}\left[a+\left(\frac{2 \beta}{V_{2}}-b\right) \sigma_{21}\right] & 0 \\
\frac{b V_{2} \mu_{2} \sigma_{12}}{V_{1}} & -b \mu_{2}
\end{array}\right], \\
p_{3}=b \mu_{2}-\mu_{1}\left[a+\sigma_{21}\left(\frac{2 \beta}{V_{2}}-b\right)\right], \\
q_{3}=\frac{b \mu_{1} \mu_{2}\left(b \sigma_{21} V_{2}-a V_{2}-2 \beta \sigma_{21}\right)}{V_{2}} .
\end{gathered}
$$


The stability condition of equilibrium point $\mathrm{O}_{3}\left(0, b V_{2}\right)$ is $\sigma_{21}>a V_{2} /\left(b V_{2}-2 \beta\right)$.

Condition $\sigma_{21}>a V_{2} /\left(b V_{2}-2 \beta\right)$ shows that message 2 is more competitive than message 1 . This just means that people accepted message 2 even though they should have believed message 1 . Therefore, during the process of competition, message 2 can survive and reach a maximum speed $V_{2}$, while message 1 will eventually become extinct finally.

3.3. Dynamics. Assume that the conditions in (25) are satisfied; the two messages in system (12) can coexist. The isocline $L_{1}$ of message 1 can be subdivided into $L_{11}$ and $L_{12}$ as follows:

$$
\begin{gathered}
L_{11}: a+\sigma_{21} \frac{v_{2}}{V_{2}}-\frac{v_{1}}{V_{1}}, \quad 0<v_{2}<\beta, \\
L_{12}: a+2 \sigma_{21} \frac{\beta}{V_{2}}-\frac{v_{1}}{V_{1}}-\sigma_{21} \frac{v_{2}}{V_{2}}, \quad v_{2}>\beta,
\end{gathered}
$$

where $L_{1}$ is smoothed in a small neighborhood of the vertex $D_{1}\left(a V_{1}+\beta \sigma_{21} V_{1} / V_{2}, \beta\right)$ as shown in Figure 1. Similarly, we divide the isocline $L_{2}$ of message 2 into $L_{21}$ and $L_{22}$ as follows:

$$
\begin{gathered}
L_{21}: b+\sigma_{12} \frac{v_{1}}{V_{1}}-\frac{v_{2}}{V_{2}}, \quad 0<v_{1}<\alpha, \\
L_{22}: b+2 \sigma_{12} \frac{\alpha}{V_{1}}-\frac{v_{2}}{V_{2}}-\sigma_{12} \frac{v_{1}}{V_{1}}, \quad v_{1}>\alpha,
\end{gathered}
$$

where $L_{2}$ is smoothed in a small neighborhood of the vertex $D_{2}\left(\alpha, b V_{2}+\alpha \sigma_{12} V_{2} / V_{1}\right)$ as shown in Figure 1.

While the line segments $L_{11}$ and $L_{21}$ are restricted to the intervals $\left(a V_{1}, a V_{1}+\beta \sigma_{21} V_{1} / V_{2}\right)$ and $(0, \alpha)$, respectively, they are impossible to intersect in the interval $\alpha \leq a V_{1}$. Then there is a maximum of three isolated positive equilibrium points in system (12). As shown in Figure 1, let $K_{1}\left(k_{11}, k_{12}\right)$ represent the intersection of $L_{11}$ and $L_{22}$, let $K_{2}\left(k_{21}, k_{22}\right)$ represent the intersection of $L_{21}$ and $L_{12}$, and let $K_{3}\left(k_{31}, k_{32}\right)$ represent the intersection of $L_{12}$ and $L_{22}$. Then we have

$$
\begin{aligned}
& k_{11}=\frac{V_{1}\left(a+b \sigma_{21}\right)+2 \alpha \sigma_{12} \sigma_{21}}{\sigma_{12} \sigma_{21}+1}, \\
& k_{12}=\frac{V_{2}\left(V_{1}\left(b-a \sigma_{12}\right)+2 \alpha \sigma_{12}\right)}{\left(\sigma_{12} \sigma_{21}+1\right) V_{1}}, \\
& k_{21}=\frac{V_{1}\left(V_{2}\left(a-b \sigma_{21}\right)+2 \beta \sigma_{21}\right)}{\left(\sigma_{12} \sigma_{21}+1\right) V_{2}}, \\
& k_{22}=\frac{V_{2}\left(a \sigma_{12}+b\right)+2 \beta \sigma_{12} \sigma_{21}}{\sigma_{12} \sigma_{21}+1}, \\
& k_{31}=\frac{V_{1}\left(V_{2}\left(b \sigma_{21}-a\right)-2 \beta \sigma_{21}\right)+2 \alpha \sigma_{12} \sigma_{21} V_{2}}{\left(\sigma_{12} \sigma_{21}-1\right) V_{2}}, \\
& k_{32}=\frac{V_{1}\left(V_{2}\left(a \sigma_{12}-b\right)+2 \beta \sigma_{12} \sigma_{21}\right)-2 \alpha \sigma_{12} V_{2}}{\left(\sigma_{12} \sigma_{21}-1\right) V_{1}} .
\end{aligned}
$$

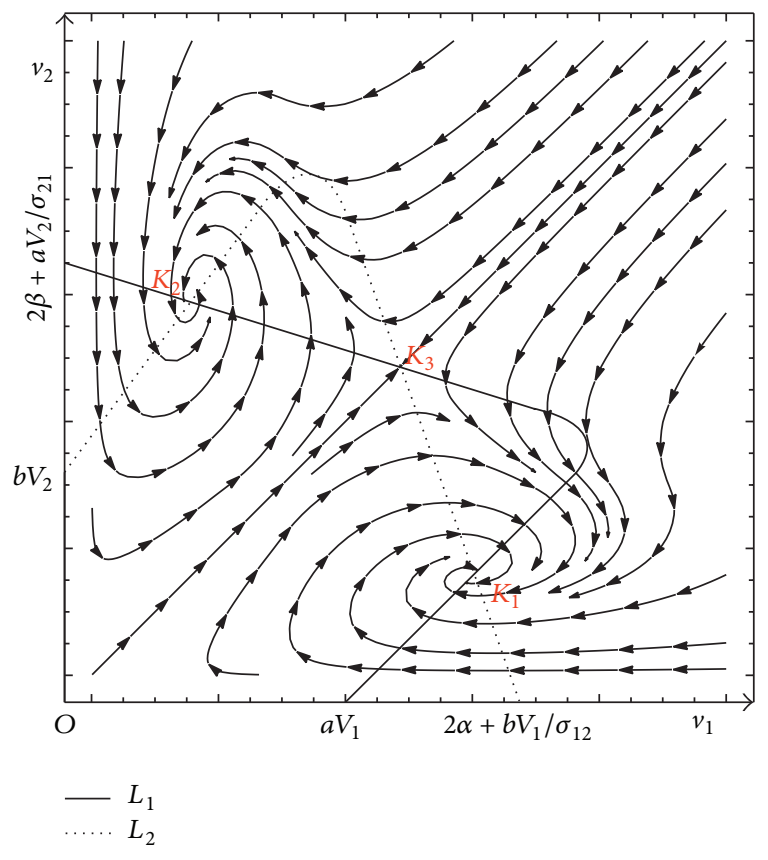

Figure 3: Three positive equilibria $K_{1}, K_{2}$, and $K_{3}$.

Suppose that $G_{1}$ and $G_{2}$ represent the slope of the lines $L_{12}$ and $L_{22}$, respectively. Suppose that $G$ denotes the slope of the line $D_{1} D_{2}$. Then we have

$$
\begin{aligned}
G_{1} & =-\frac{V_{2}}{V_{1} \sigma_{21}}<0, \\
G_{2} & =-\frac{V_{2} \sigma_{12}}{V_{1}}<0, \\
G & =\frac{V_{2}\left(b V_{2} V_{1}+\alpha \sigma_{12} V_{2}-\beta V_{1}\right)}{V_{1}\left(a V_{1} V_{2}-\alpha V_{2}+\beta \sigma_{21} V_{1}\right)}<0 .
\end{aligned}
$$

Dynamics of system (12) can be displayed in five cases, while we discuss the situation of $\left|G_{1}\right|<\left|G_{2}\right|$ in cases 1-4 and that of $\left|G_{1}\right|>\left|G_{2}\right|$ in case 5 .

(i) Case $1\left(\left|G_{1}\right|<|G|<\left|G_{2}\right|\right)$. In this case, there are three positive equilibria $K_{1}, K_{2}$, and $K_{3}$ as shown in Figure 3. The Jacobian matrix of system (12) at $K_{1}$ is

$$
M\left(K_{1}\right)=\left[\begin{array}{cc}
-\frac{k_{11} \mu_{1}}{V_{1}} & \frac{k_{11} \mu_{1} \sigma_{21}}{V_{2}} \\
-\frac{k_{12} \mu_{2} \sigma_{12}}{V_{1}} & -\frac{k_{12} \mu_{2}}{V_{2}}
\end{array}\right]
$$

then $p\left(M\left(K_{1}\right)\right)=k_{11} \mu_{1} / V_{1}+\mu_{2} k_{12} / V_{2}>0$ and $q\left(M\left(K_{1}\right)\right)=$ $k_{11} k_{12} \mu_{1} \mu_{2}\left(\sigma_{12} \sigma_{21}+1\right) / V_{1} V_{2}>0$. That is, $K_{1}$ is asymptotically stable. Then we obtain that the stability condition of equilibrium point $K_{1}$ is

$$
\begin{aligned}
0<\sigma_{12}< & \frac{\mu_{1} V_{1}\left(\lambda_{2}-\mu_{2}\right)}{\mu_{2}\left(2 \alpha \mu_{1}+\lambda_{1} V_{1}-\mu_{1} V_{1}\right)}, \\
& \sigma_{21}>0, \quad V_{1}>\frac{2 \alpha \mu_{1}}{\mu_{1}-\lambda_{1}} .
\end{aligned}
$$




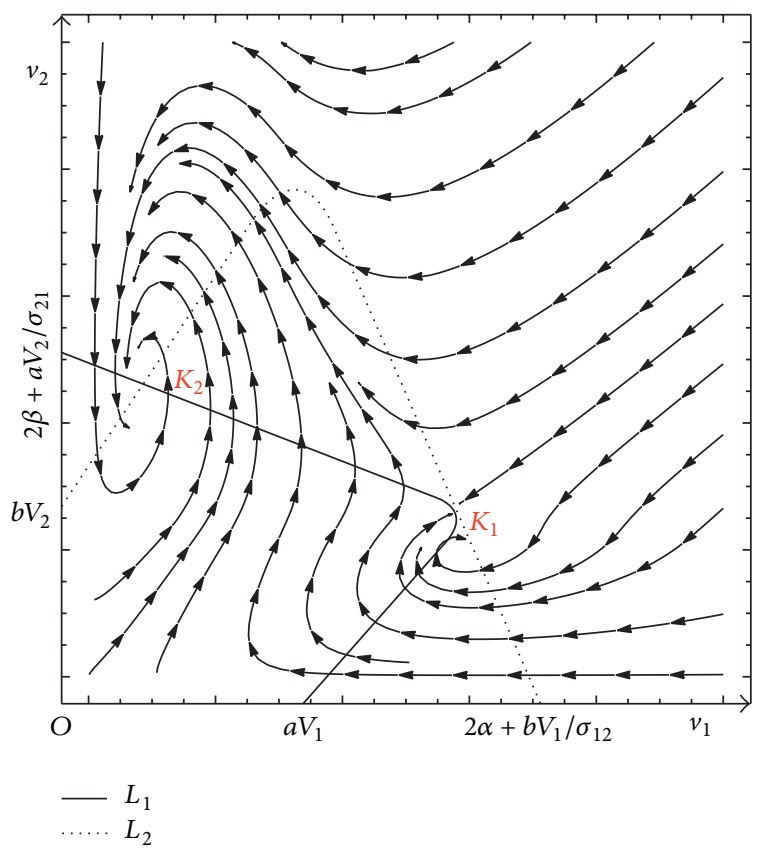

FIgURE 4: Two positive equilibria $K_{1}$ and $K_{2}$.

Condition $0<\sigma_{12}<\mu_{1} V_{1}\left(\lambda_{2}-\mu_{2}\right) / \mu_{2}\left(2 \alpha \mu_{1}+\lambda_{1} V_{1}-\right.$ $\left.\mu_{1} V_{1}\right)$ means that message 1 is less competitive than message 2 . When (33) are satisfied, both message 1 and message 2 coexist, and the speed of message 1 and message 2 will tend to reach a nonzero finite value.

Similarly, The Jacobian matrix of system (12) at $K_{2}$ is

$$
M\left(K_{2}\right)=\left[\begin{array}{cc}
-\frac{k_{21} \mu_{1}}{V_{1}} & -\frac{k_{21} \mu_{1} \sigma_{21}}{V_{2}} \\
\frac{k_{22} \mu_{2} \sigma_{12}}{V_{1}} & -\frac{k_{22} \mu_{2}}{V_{2}}
\end{array}\right]
$$

then the $p\left(M\left(K_{2}\right)\right)=k_{21} \mu_{1} / V_{1}+k_{22} \mu_{2} / V_{2}>0$ and $q\left(M\left(K_{2}\right)\right)=k_{21} k_{22} \mu_{1} \mu_{2}\left(\sigma_{12} \sigma_{21}+1\right) / V_{1} V_{2}>0$. That is, $K_{2}$ is asymptotically stable. Then we obtain that the stability condition of equilibrium point $K_{2}$ is

$$
\begin{array}{r}
0<\sigma_{21}<\frac{\mu_{2} V_{2}\left(\lambda_{1}-\mu_{1}\right)}{\mu_{1}\left(2 \beta \mu_{2}+\lambda_{2} V_{2}-\mu_{2} V_{2}\right)}, \\
\sigma_{12}>0, \quad V_{2}>\frac{2 \beta \mu_{2}}{\mu_{2}-\lambda_{2}} .
\end{array}
$$

The condition $0<\sigma_{21}<\mu_{2} V_{2}\left(\lambda_{1}-\mu_{1}\right) / \mu_{1}\left(2 \beta \mu_{2}+\lambda_{2} V_{2}-\right.$ $\mu_{2} V_{2}$ ) means that message 2 is less competitive than message 1 . When (35) are satisfied, both message 1 and message 2 coexist, and their speed will change over time to reach a stable state.

The Jacobian matrix of system (12) at $K_{3}$ is

$$
M\left(K_{3}\right)=\left[\begin{array}{cc}
-\frac{k_{31} \mu_{1}}{V_{1}} & -\frac{k_{31} \mu_{1} \sigma_{21}}{V_{2}} \\
-\frac{k_{32} \mu_{2} \sigma_{12}}{V_{1}} & -\frac{k_{32} \mu_{2}}{V_{2}}
\end{array}\right]
$$

then the $p\left(M\left(K_{3}\right)\right)=k_{31} \mu_{1} / V_{1}+k_{32} \mu_{2} / V_{2}>0$ and $q\left(M\left(K_{3}\right)\right)=k_{31} k_{32} \mu_{1} \mu_{2}\left(1-\sigma_{12} \sigma_{21}\right) / V_{1} V_{2}$. By $\left|G_{1}\right|<\left|G_{2}\right|$,

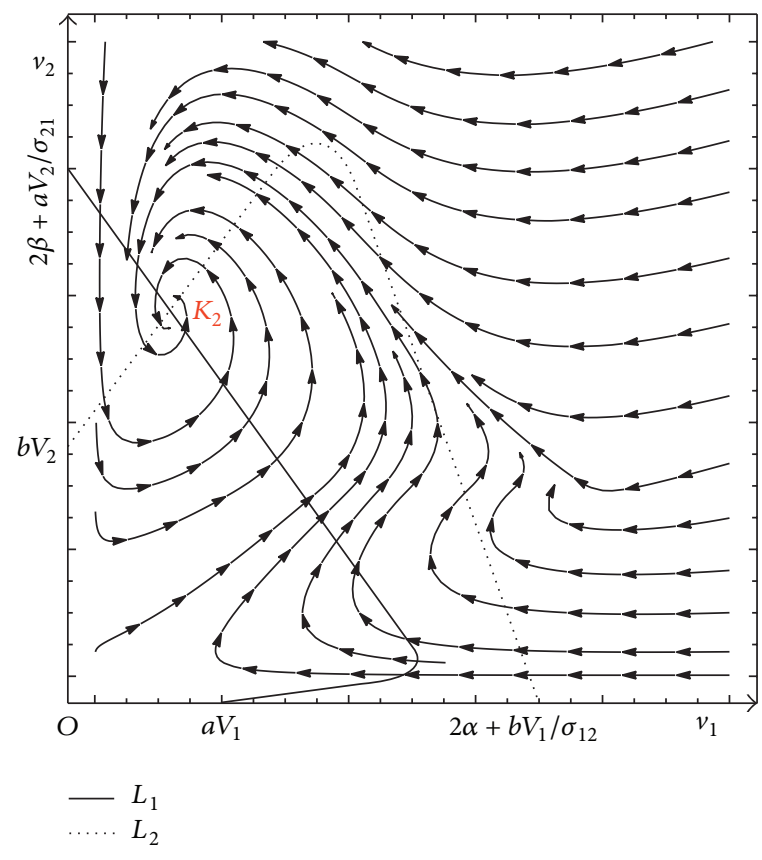

FIgURE 5: The unique positive equilibrium $K_{2}$.

we have $\sigma_{12} \sigma_{21}>1$, and $q\left(M\left(K_{3}\right)\right)<0$. It means that $K_{3}$ is a saddle point. In int $R_{+}^{2}$, all orbits but $K_{3}$ and the separatrixes of $K_{3}$ converge to equilibria $K_{1}$ and $K_{2}$ as shown in Figure 3.

(ii) Case $2\left(\left|G_{1}\right|<|G|=\left|G_{2}\right|\right)$. As can be seen in Figure 4, there are two positive equilibrium points $K_{1}$ and $K_{2}$. It is important to note that, however, $K_{1}$ and $K_{3}$ in Figure 3 coincide. Similar to the analysis in case $1, K_{2}$ is asymptotically stable, and the stability condition of equilibrium point $K_{2}$ satisfies (35).

Since $p\left(M\left(K_{1}\right)\right)=k_{11} \mu_{1} / V_{1}+\mu_{2} k_{12} / V_{2}>0$ and $q\left(M\left(K_{1}\right)\right)=0$, it can be determined that $K_{1}$ is a saddle node by the saddle node rule [26]. According to Theorem 1, we can draw the dynamics of system (12) as shown in Figure 4 . The same approach also applies to the case $\left|G_{1}\right|=|G|<\left|G_{2}\right|$; the difference is that $K_{1}$ is asymptotically stable and $K_{2}$ is a saddle node equilibrium, and all orbits converge to equilibria $K_{1}$ in int $R_{+}^{2}$ except $K_{2}$ and the separatrixes of $K_{2}$.

(iii) Case $3\left(\left|G_{1}\right| \leq\left|G_{2}\right|<|G|\right)$. In case 3, there is only one positive equilibrium point $K_{2}$. By Theorem $1, K_{2}$ is asymptotically stable as shown in Figure 5, and the stability condition of equilibrium point $K_{2}$ satisfies (33). Similar discussions can be given for the case $|G|<\left|G_{1}\right| \leq\left|G_{2}\right|$ in which $K_{1}$ is asymptotically stable.

(iv) Case $4\left(\left|G_{1}\right|=\left|G_{2}\right|=|G|\right)$. In this case, the isoclines $L_{12}$ and $L_{22}$ coincide, and all the positive equilibrium points distribute on the line segment $D_{1} D_{2}$. According to Theorem 1 , in int $R_{+}^{2}$, all orbits of system (12) converge to the line segment as shown in Figure 6.

(v) Case $5\left(\left|G_{2}\right|<\left|G_{1}\right|\right)$. In this situation, we have similar discussions with the case 3 , and globally stable is $K_{2}$ as shown 


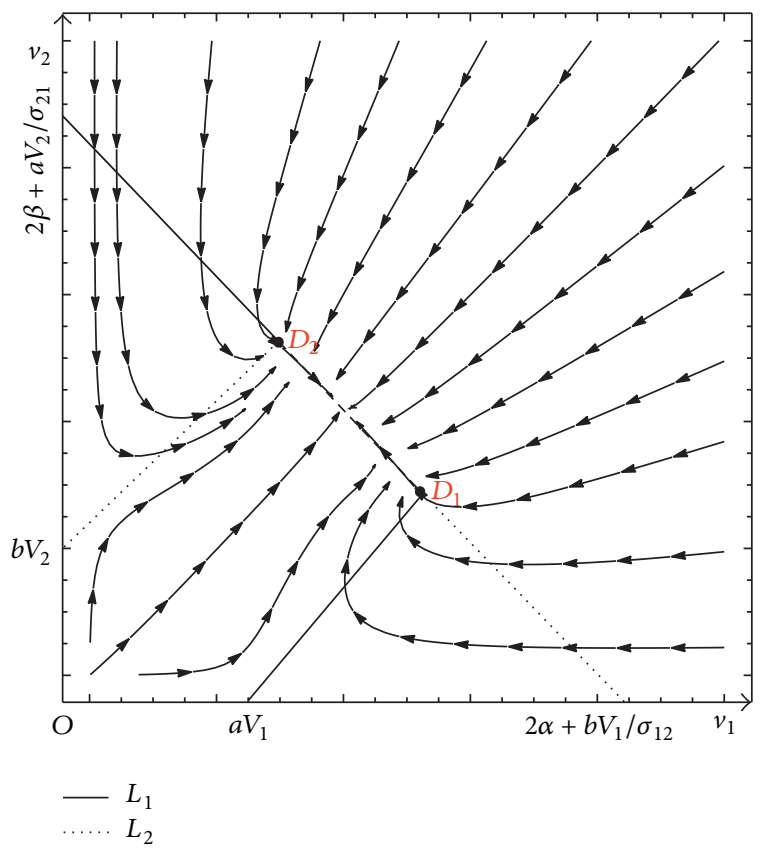

FIGURE 6: Equilibrium points on the line segment $D_{1} D_{2}$.

in Figure 5. The same principle applies to the condition of $|G|<\left|G_{2}\right|<\left|G_{1}\right|$ that $K_{1}$ is globally stable. In the case of $\left|G_{2}\right| \leq|G|<\left|G_{1}\right|$ or $\left|G_{2}\right|<|G| \leq\left|G_{1}\right|$, there is a unique positive globally stable point $K_{3}$ of system (12) in Figure 7.

\section{Numerical Simulation}

To better understand the proposed model, we carry out computer simulations with real data from Sina Weibo, a Chinese microblog. Our data collected by a crowd sourcing Weibo visual analytic system [27], and the interval time is from March 31th, 2014, to May 30th, 2014. We extract two messages published by famous Chinese actors Wen Zhang and Ma Yili about their marriage. In Sina Weibo, Wen Zhang has 53706926 fans, ranked in 7th place and Ma Yili has 34 759267 fans, ranked in 35th place. At midnight of March 31th, Wen Zhang apologised to his families and devoted fans, and then his wife Ma Yili forgives him. For their action in saving marriage, amount of their fans reposted the two messages. As the repost actions have occurred mainly before 14:00, we specify a unit time in 15 minutes to statistical analysis among 11:00-14:00 in March 31th. In order to explore the dynamics process with varied parameters, we put the observations in our model and then further study the influence of the competition coefficient $\left(\sigma_{12}\right.$ and $\left.\sigma_{21}\right)$ in case of messages propagation with low (or high) critical speed ( $\alpha$ and $\beta$ ).

We first consider the case that both messages have less competitive $\sigma_{12}<b V_{1} /\left(a V_{1}-2 \alpha\right)$ and $\sigma_{21}<a V_{2} /\left(b V_{2}-2 \beta\right)$ at low critical speed. Figure $8($ a) shows the evolutionary trend of message 1 and message 2 over time. We find that the speed of the two messages can be viewed as a nonmonotonic function, and the velocity curve of message 1 can be divided into three distinctly different stages. In the initial stage, message 1 appears in OSNs and then attracts more and more users,

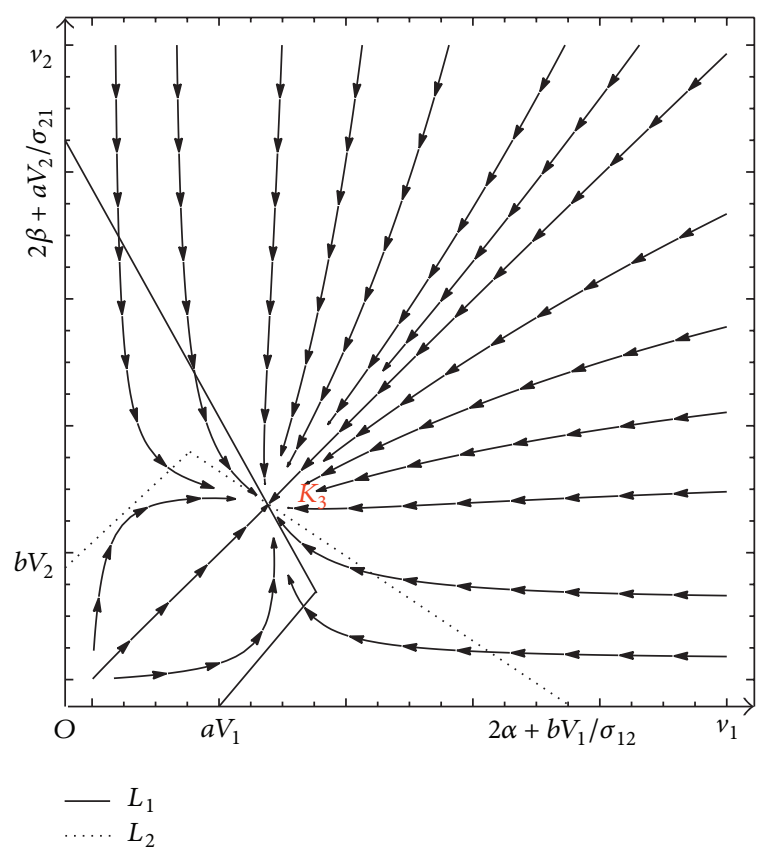

FIGURE 7: The unique positive equilibrium $K_{3}$.

leading to rapid spread of message 1 . However, message 1 descends slowly followed by the end of initial stage, because of the natural dying out of its own as well as the competition coming from message 2. Specifically, since message 1 is less competitive than message 2 , the growth of message 2 hinders the spread of message 1 and message 1 will continuously decay due to the competition. Finally, message 1 arrives at its equilibrium and then converges to a constant value. The evolution process of message 2 is similar to message 1 . After a slow growth in the initial stage, message 2 spreads rapidly and then slows down till a steady state is reached.

The change trends of message 1 and message 2 can be observed from Figure 8(b). At the beginning, the two messages grow quickly and then slow down and converge to a nonzero constant. This process reflects that, with less competitive and low critical speed, message 1 and message 2 will cooperate with each other in the propagation. Both of them can attract a certain amount of users, and they can coexist in a period of time.

In the case of $\sigma_{12}<b V_{1} /\left(a V_{1}-2 \alpha\right)$ and $\sigma_{21}>$ $a V_{2} /\left(b V_{2}-2 \beta\right)$, the message which is more competitive than the other will survive at the final state. As shown in Figure 9(a), along with the increasing number of people who have heard message 2 , the speed of message 1 increases at the beginning and then decreases rapidly. The main reason for this phenomenon is that the two messages have cooperated with each other when their speed is lower than the critical speed at the beginning, and then competed against each other when their speed is higher than the critical speed after the initial phase. Finally, only the more competitive message $\left(\sigma_{21}^{9(a)} \gg \sigma_{12}^{9(a)}\right)$ survived in the system. When $\sigma_{12}>$ $b V_{1} /\left(a V_{1}-2 \alpha\right)$ and $\sigma_{21}<a V_{2} /\left(b V_{2}-2 \beta\right)$, we can get a similar conclusion that message 2 will vanish at stable state. That is, the more competitive message can draw the users attention 


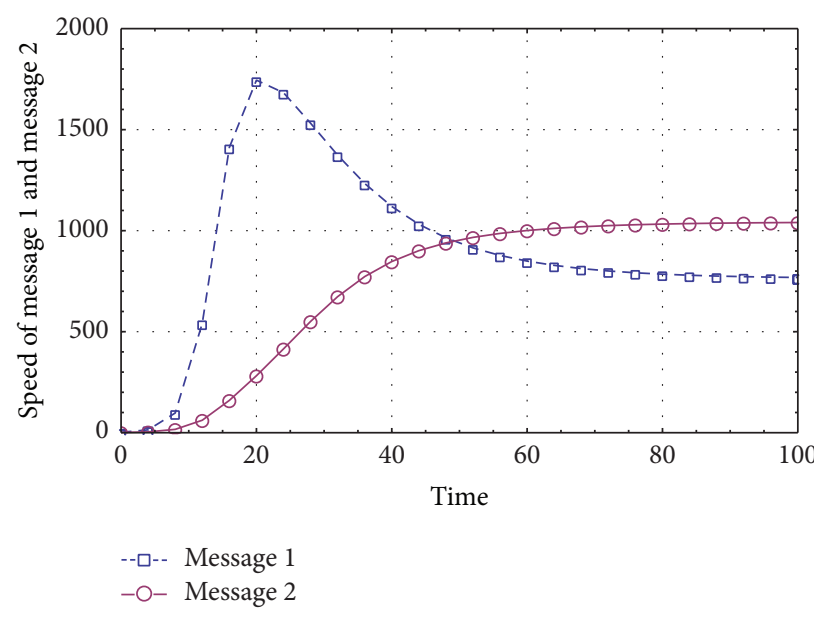

(a)

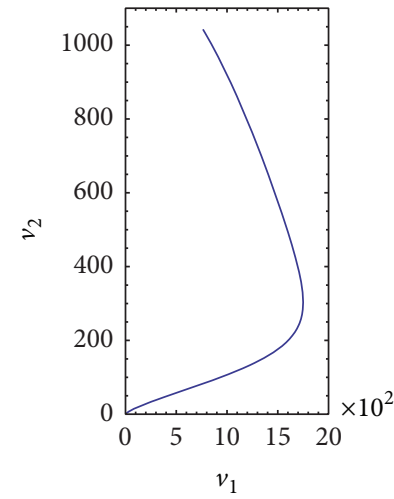

(b)

FIGURE 8: (a) $v_{1}$ and $v_{2}$ as a function of $t$, with $v_{1}(0)=2, v_{2}(0)=1, V_{1}=3000, \alpha=80, \sigma_{12}^{8(a)}=0.6, \mu_{1}=0.7, \lambda_{1}=0.2, V_{2}=1800, \beta=20$, $\sigma_{21}^{8(a)}=0.8, \mu_{2}=0.5$, and $\lambda_{2}=0.15$. (b) The development trend of relationship between message 1 and message 2 .

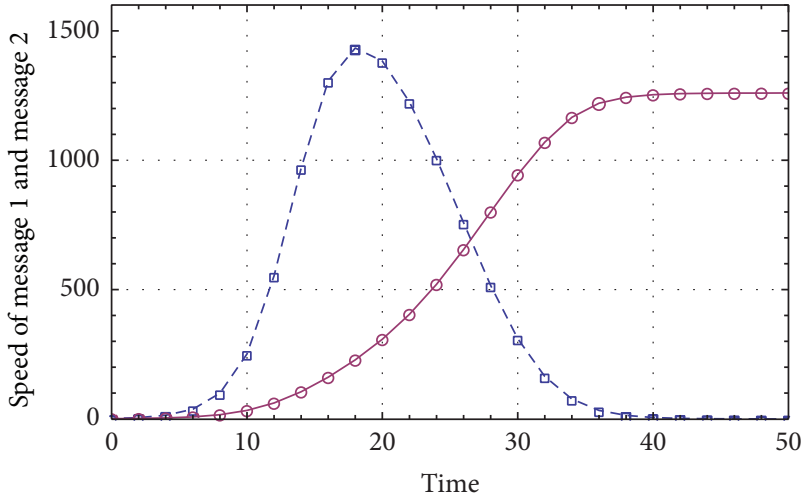

--ם-- Message 1

-O- Message 2

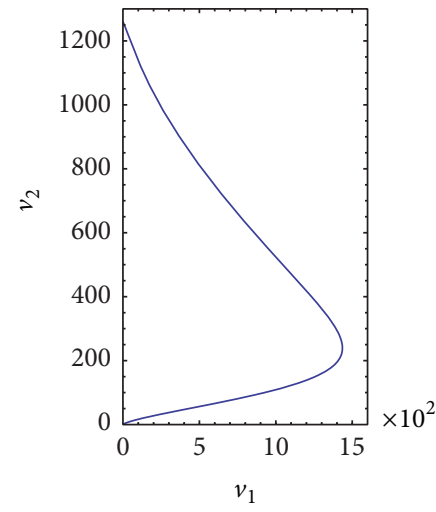

(b)

Figure 9: (a) $v_{1}$ and $v_{2}$ as a function of $t$, with $v_{1}(0)=2, v_{2}(0)=1, V_{1}=3000, \alpha=80, \sigma_{12}^{9(a)}=0.6, \mu_{1}=0.7, \lambda_{1}=0.2, V_{2}=1800, \beta=20$, $\sigma_{21}^{9(a)}=2, \mu_{2}=0.5$, and $\lambda_{2}=0.15$. (b) The development trend of relationship between message 1 and message 2 .

and spread widely while the message with lesser competitive is difficult to attract users and vanished after a while.

The change trends of message 1 and message 2 in Figure $9(\mathrm{~b})$ is similar to that in Figure $8(\mathrm{~b})$. The difference is that only message 2 can survive when a final state is reached. Under the constraint condition of parameters $\sigma_{21}^{9(a)} \gg \sigma_{21}^{8(a)}$, we can make a conclusion that message 2 attracts amount of users from message 1 at low critical speed and spreads widely in online social networks.

As shown in Figure 10(a), under the condition of $\sigma_{12}>$ $b V_{1} /\left(a V_{1}-2 \alpha\right)$ and $\sigma_{21}>a V_{2} /\left(b V_{2}-2 \beta\right)$, only message 1 survives and message 2 vanishes in steady state, while they coexist with each other at the final stage in Figure 10(b). It can be inferred that in strong competitive environment, the high critical speed promotes the coexistence of the two messages. At the same time, although restricted by $\sigma_{21}<\sigma_{12}$, the velocity values of both message 1 and message 2 in Figure 10(b) are greater than that in Figure 10(a); it can be concluded that when the two messages are both competitive, they will benefit from the high critical speed.

Generally, more competitive messages disseminate more rapidly than less competitive messages over network. Unfortunately, the situation is often not the case. It can be observed that under the conditions with the same $\sigma_{21}$, message 2 can survive and reach its maximum speed in Figure 9(a), but it vanished finally in Figure 10(a). The difference exists because the competition coefficient of message $1\left(\sigma_{12}\right)$ is considerably larger in Figure 10(a) than that in Figure 9(a) $\left(\sigma_{12}^{10(a)} \gg\right.$ $\left.\sigma_{12}^{9(a)}\right)$. It means that the advantage of the prior competitive message will quickly disappear as the competitiveness gap narrowed. Therefore, under the condition of low critical speed, it is necessary to adopt Tit-for-Tat-like strategy to 


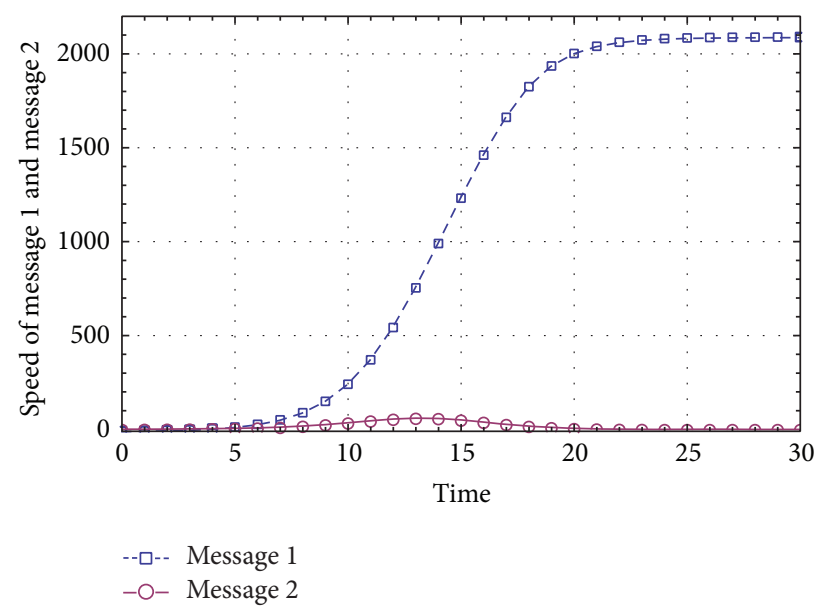

(a)

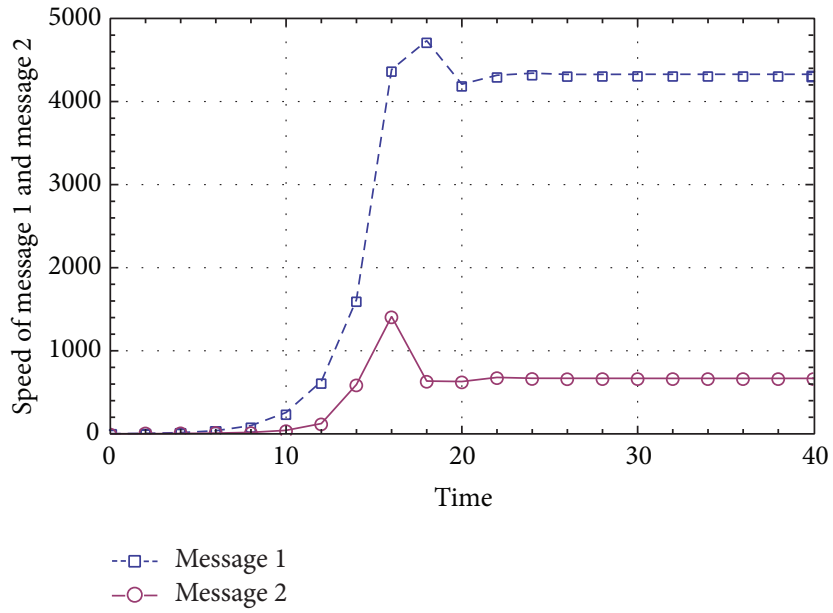

(b)

FIGURE 10: Positive or negative effects for different critical speed with $v_{1}(0)=2, v_{2}(0)=1, V_{1}=3000, \sigma_{12}^{10(a)}=3, \mu_{1}=0.7, \lambda_{1}=0.2, V_{2}=1800$, $\sigma_{21}^{10(a)}=2, \mu_{2}=0.5$, and $\lambda_{2}=0.15$. (a) $v_{1}$ and $v_{2}$ as a function of $t$, with $\alpha=80, \beta=20$. (b) $v_{1}$ and $v_{2}$ as a function of $t$, with $\alpha=2000$, $\beta=1200$.

improve their competitiveness when there already exists a competitive message in online social network.

\section{Conclusions}

The majority of studies on information propagation have focused on a single contagion spreading through the social network without considering the influence of other contagions. In this paper, we have explored the information spreading mechanism impacted by cooperative and competitive effectiveness during the process of the propagation of two types of messages over networks. We developed a new OSN information propagation model based on LotkaVolterra models to demonstrate the dynamics of a specific cooperation-competition system of these two messages. The stability of the system was proved by the differential equations and the phase trajectory of the stability theory. The results of stability analysis further indicated that the differential equations admit no periodic orbit, and the system can reach steady state in certain condition.

We proved that two types of messages usually cannot spread together peacefully, and only one type message survives till final state. Nevertheless, it produced stable points in the condition of high (or low) critical speed, which shows that it is possible for the coexistence of both messages in competitive propagation of different contagions. Using the real data collected from Sina Weibo, the results validated the theoretical analysis and presented another interesting conclusion that the messages will benefit from the high critical speed when they are both competitive. If there already is a competitive message in OSN, it is wise to adopt a Tit-forTat strategy.

As the great massive data are generated every moment in OSN, the rapid progress of information technology provides us with an easy way to collect data and observe the phenomenon that various types of information flow in the form of cascades on Twitter or other OSNs. Naturally, the proposed dynamic information propagation model should be tested and applied in real OSN with large topology network and vast numbers of the nodes and connections. These issues will be addressed in future research.

\section{Conflict of Interests}

The authors declare that there is no conflict of interests regarding the publication of this paper.

\section{Acknowledgments}

This work was partially supported by the National Nature Science Foundation of China (no. 71271186), Education Ministry Fund of Humanities and Social Science (no. 12YJA630191), Natural Science Foundation of Hebei (no. G2013203237), and Brazilian National Council for Scientific and Technological Development-CNPq (no. 304903/2013-2).

\section{References}

[1] W. W. Wu, "The cooperation-competition model for the hot topics of Chinese microblogs," Applied Mechanics and Materials, vol. 380-384, pp. 2724-2727, 2013.

[2] J. C. Bosley, N. W. Zhao, S. Hill et al., "Decoding twitter: Surveillance and trends for cardiac arrest and resuscitation communication," Resuscitation, vol. 84, no. 2, pp. 206-212, 2013.

[3] L. Weigang, E. F. O. Sandes, J. Zheng, A. C. M. A. Melo, and L. Uden, "Querying dynamic communities in online social networks," Journal of Zhejiang University Science C, vol. 15, no. 2, pp. 81-90, 2014.

[4] X. Zhao and J. Wang, "Dynamical model about rumor spreading with medium," Discrete Dynamics in Nature and Society, vol. 2013, Article ID 586867, 9 pages, 2013.

[5] D. J. Daley and D. G. Kendall, "Epidemics and Rumours [49]," Nature, vol. 204, no. 4963, p. 1118, 1964. 
[6] D. J. Daley and D. G. Kendall, "Stochastic rumours," Journal of the Institute of Mathematics and Its Applications, vol. 1, pp. 4255, 1965.

[7] D. P. Maki and M. Thompson, Mathematical Models and Applications: With Emphasis on the Social, Life, and Management Sciences, Prentice Hall, Englewood Cliffs, NJ, USA, 1973.

[8] J. R. C. Piqueira, "Rumor propagation model: an equilibrium study," Mathematical Problems in Engineering, vol. 2010, Article ID 631357, 7 pages, 2010.

[9] D. H. Zanette, "Dynamics of rumor propagation on small-world networks," Physical Review E, vol. 65, no. 4, Article ID 041908, 9 pages, 2002.

[10] Y. Moreno, M. Nekovee, and A. Vespignani, "Efficiency and reliability of epidemic data dissemination in complex networks," Physical Review E, vol. 69, Article ID 055101(R), 2004.

[11] Y. Moreno, M. Nekovee, and A. F. Pacheco, "Dynamics of rumor spreading in complex networks," Physical Review E: Statistical, Nonlinear, and Soft Matter Physics, vol. 69, no. 6, Article ID 066130, 2004.

[12] J. Zhou, Z. Liu, and B. Li, "Influence of network structure on rumor propagation," Physics Letters A, vol. 368, no. 6, pp. 458463, 2007.

[13] M. Nekovee, Y. Moreno, G. Bianconi, and M. Marsili, "Theory of rumour spreading in complex social networks," Physica A, vol. 374, no. 1, pp. 457-470, 2007.

[14] M. Granovetter, "Threshold models of collective behavior," The American Journal of Sociology, pp. 1420-1443, 1978.

[15] D. Kempe, J. Kleinberg, and É. Tardos, "Maximizing the spread of influence through a social network," in Proceedings of the 9th ACM SIGKDD International Conference on Knowledge Discovery and Data Mining (KDD '03), pp. 137-146, New York, NY, USA, August 2003.

[16] D. Trpevski, W. K. S. Tang, and L. Kocarev, "Model for rumor spreading over networks," Physical Review E, vol. 81, no. 5, Article ID 056102, 2010.

[17] Z. Z. Liu, W. Xing-Yuan, and W. Mao-Ji, "Competition between two kinds of information among random-walking individuals," Chinese Physics B, vol. 21, no. 4, Article ID 048902, 6 pages, 2012.

[18] X. T. Wei, N. Valler, B. A. Prakash, I. Neamtiu, M. Faloutsos, and C. Faloutsos, "Competing memes propagation on networks: a case study of composite networks," ACM SIGCOMM Computer Communication Review, vol. 42, no. 5, pp. 5-12, 2012.

[19] Y. B. Wang, G. X. Xiao, and J. Liu, "Dynamics of competing ideas in complex social systems," New Journal of Physics, vol. 14, no. 1, Article ID 013015, 22 pages, 2012.

[20] L. Weng, A. Flammini, A. Vespignani, and F. Menczer, "Competition among memes in a world with limited attention," Scientific Reports, vol. 2, article 335, 8 pages, 2012.

[21] S. A. Myers and J. Leskovec, "Clash of the contagions: cooperation and competition in information diffusion," in Proceedings of the 12th IEEE International Conference on Data Mining (ICDM '12), pp. 539-548, Los Alamitos, CA, USA, December 2012.

[22] L. Lü, D. Chen, and T. Zhou, "The small world yields the most effective information spreading," New Journal of Physics, vol. 13, Article ID 123005, 2011.

[23] M. J. Hernandez and I. Barradas, "Variation in the outcome of population interactions: bifurcations and catastrophes," Journal of Mathematical Biology, vol. 46, no. 6, pp. 571-594, 2003.

[24] Z. Zhang, "Mutualism or cooperation among competitors promotes coexistence and competitive ability," Ecological Modelling, vol. 164, no. 2-3, pp. 271-282, 2003.
[25] J. Hofbauer and K. Sigmund, Evolutionary Games and Population Dynamics, Cambridge University Press, Cambridge, UK, 1998.

[26] Z. Zhang, T. Ding, W. Huang, and Z. Dong, Qualitative Theory of Differential Equations, vol. 101 of Translations of Mathematical Monographs, AMS, Providence, RI, USA, 1992.

[27] D. Ren, X. Zhang, Z. Wang, J. Li, and X. Yuan, "WeiboEvents: a crowd sourcing weibo visual analytic system," in Proceedings of the IEEE Pacific Visualization Symposium (PacificVis '14), pp. 330-334, Yokohama, Japan, March 2014. 


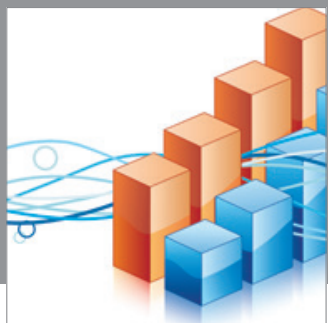

Advances in

Operations Research

mansans

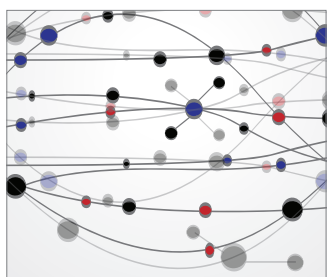

The Scientific World Journal
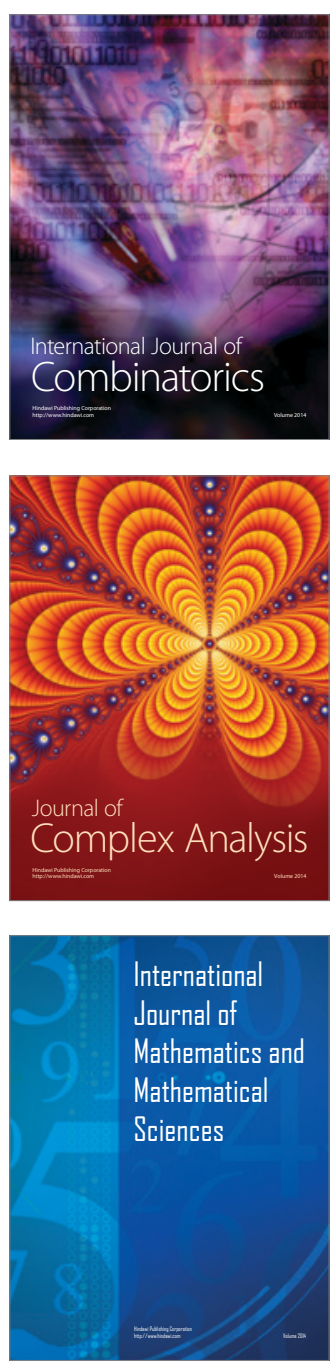
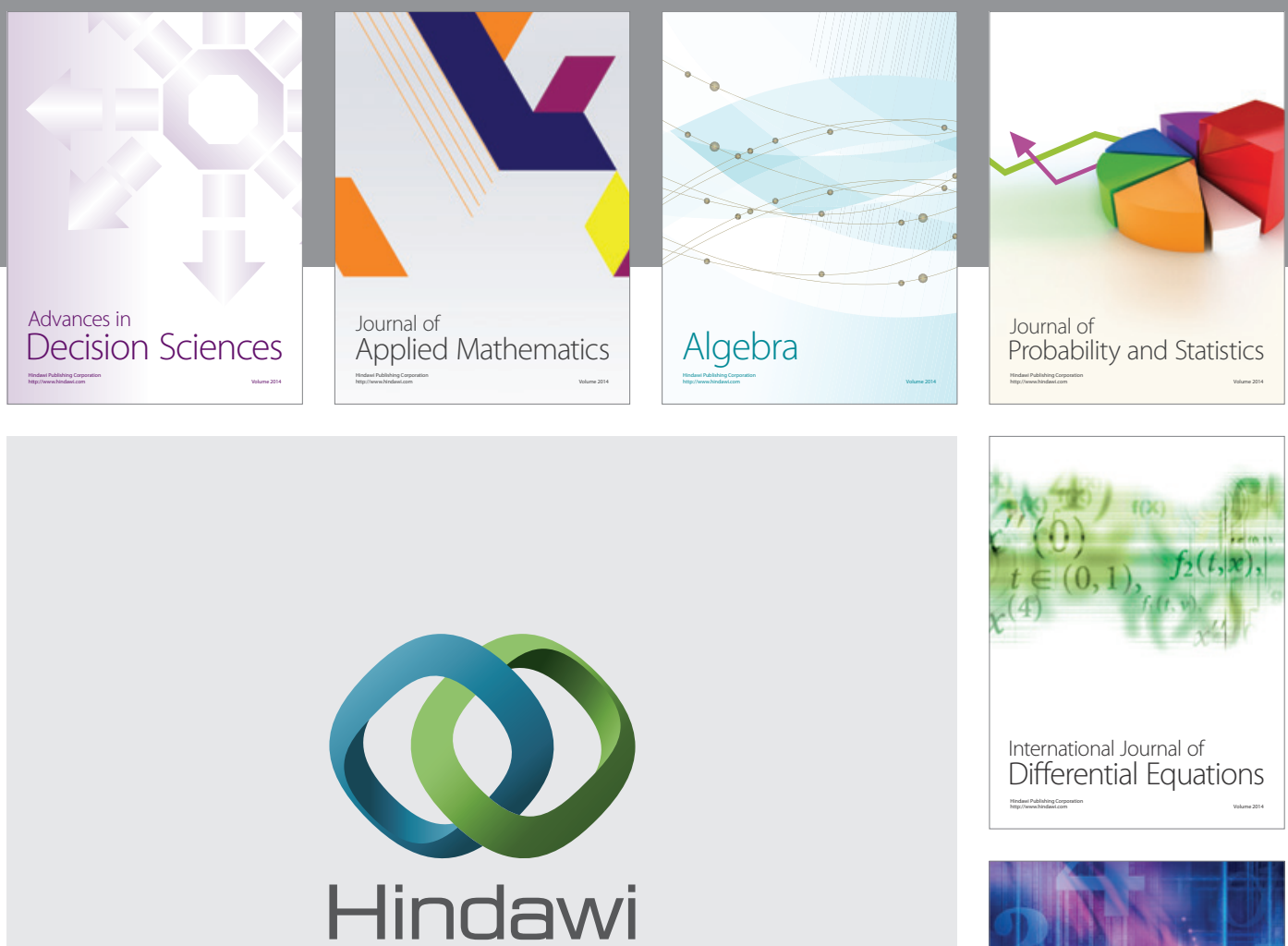

Submit your manuscripts at http://www.hindawi.com
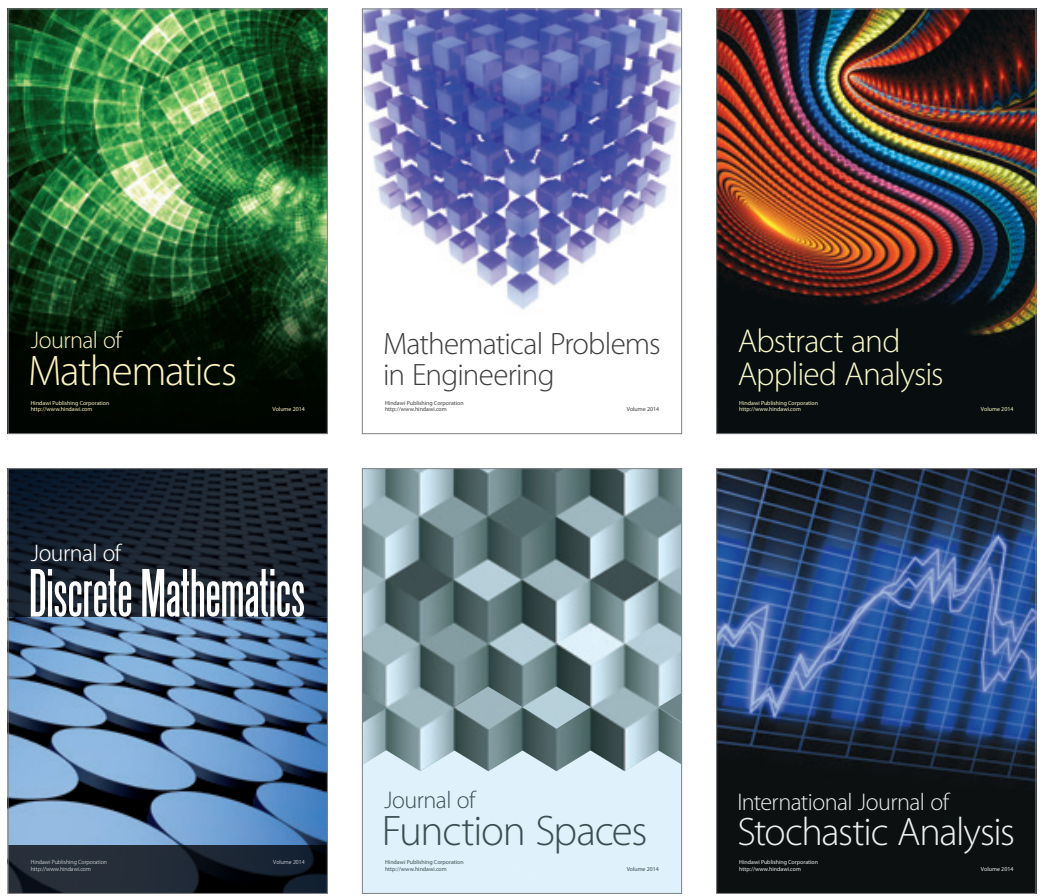

Journal of

Function Spaces

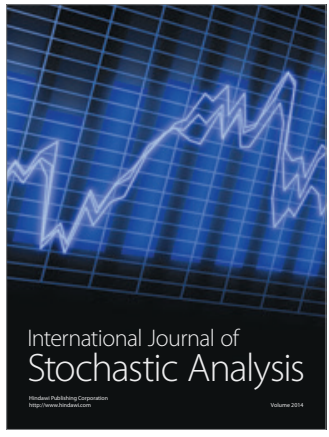

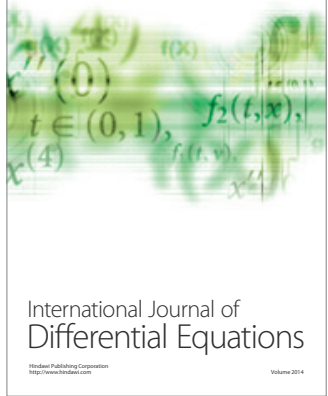
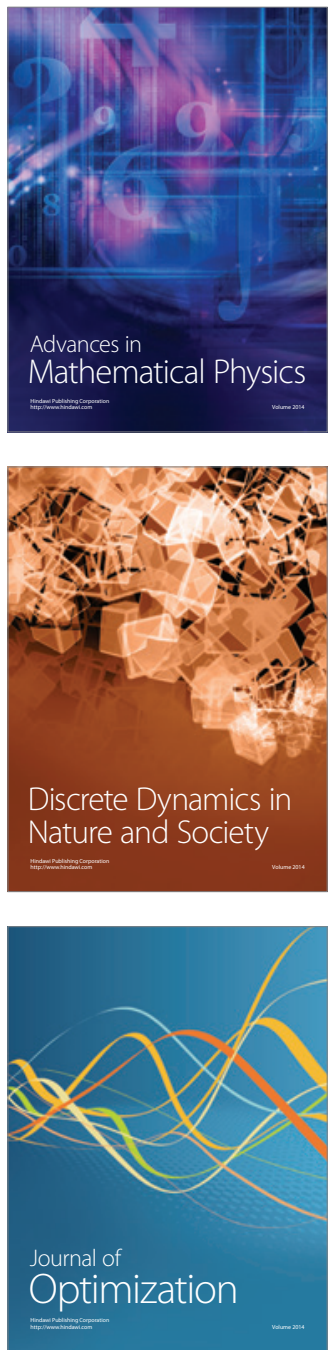